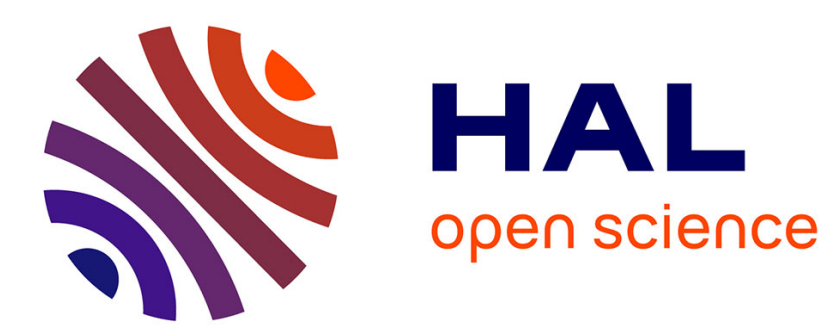

\title{
Mosaicism for oncogenic G12D KRAS mutation associated with epidermal nevus, polycyctic kidneys and rhabdomyosarcoma \\ Olivier Delattre
}

\section{- To cite this version:}

Olivier Delattre. Mosaicism for oncogenic G12D KRAS mutation associated with epidermal nevus, polycyctic kidneys and rhabdomyosarcoma. Journal of Medical Genetics, 2010, 47 (12), pp.859. 10.1136/jmg.2009.075374 . hal-00560777

\section{HAL Id: hal-00560777 https://hal.science/hal-00560777}

Submitted on 30 Jan 2011

HAL is a multi-disciplinary open access archive for the deposit and dissemination of scientific research documents, whether they are published or not. The documents may come from teaching and research institutions in France or abroad, or from public or private research centers.
L'archive ouverte pluridisciplinaire HAL, est destinée au dépôt et à la diffusion de documents scientifiques de niveau recherche, publiés ou non, émanant des établissements d'enseignement et de recherche français ou étrangers, des laboratoires publics ou privés. 


\section{Mosaicism for oncogenic G12D KRAS mutation associated with epidermal nevus,}

polycystic kidneys and rhabdomyosarcoma.

Franck Bourdeaut ${ }^{1,2,3}$, Aurélie Hérault ${ }^{3,4}$, David Gentien ${ }^{5}$, Gaëlle Pierron ${ }^{6}$, Stelly Ballet ${ }^{6}$, Stéphanie Reynaud ${ }^{6}$, Régine Paris ${ }^{7}$, Gudrun Schleiermacher ${ }^{2,3,8}$, Clarisse Baumann ${ }^{9}$, Pascale Philippe-Chomette ${ }^{10}$, Marion Gauthier-Villars ${ }^{11}$, Michel Peuchmaur ${ }^{7,12}$, François Radvanyi ${ }^{3,4}$, Olivier Delattre $e^{2,3,6}$

${ }^{1}$ CHU Nantes, Service d'oncologie pédiatrique, quai Moncousu, Nantes, 44, France

${ }^{2}$ INSERMU830, Laboratoire de biologie et génétique des cancers, 26 rue d’Ulm, Institut Curie, Paris 75248 CEDEX 5, France

${ }^{3}$ Institut Curie, Centre de recherche, 26 rue d'Ulm, 75248 Paris CEDEX5, France

${ }^{4}$ CNRS, U144, 2 rue Lhomond, Institut Curie, 75248 CEDEX 5 Paris, France

${ }^{5}$ Institut Curie, Département de transfert, 1 avenue Claude Vellefaux, 75475 Paris CEDEX10, France

${ }^{6}$ Institut Curie, Unité de génétique somatique, 26 rue d’Ulm, 75248 CEDEX 5 Paris, France

${ }^{7}$ Hôpital Robert Debré, Service d'anatomie pathologique, 48 boulevard Serrurier, 75935 Paris, CEDEX 19, France

${ }^{8}$ Institut Curie, Département de pédiatrie, 26 rue d’Ulm, 75248 CEDEX 5 Paris, France

${ }^{9}$ Hôpital Robert Debré, Service de génétique, 49 boulevard Serrurier, 75935 Paris, CEDEX 19, France

${ }^{10}$ Hôpital Robert Debré, Service de chirurgie infantile, 49 boulevard Serrurier, 75935 Paris, CEDEX 19, France

${ }^{11}$ Institut Curie, Département d'oncologie génétique, 26 rue d’Ulm, 75248 CEDEX 5 Paris, France

${ }^{12}$ Université Paris XI, Paris, France 
Corresponding author: Olivier Delattre, INSERM U830, Institut Curie, 26 rue d'Ulm 75248

PARIS CEDEX05, FRANCE

Phone : 33156246679

Fax : 33156246630

Email: Olivier.delattre@ curie.fr

Key-words: mosaicism, KRAS, rhabdomyosarcoma, epidermal nevus

Word count: 1432 
Abstract:

Epidermal nevus (EN) is a congenital disorder characterized by hyperpigmented epidermal thickening following a Blaschko's line. It is due to somatic mutations in either FGFR3 or PIK3CA in half of cases, and remains of unknown genetic origin in the other half. EN is also observed as part of complex developmental disorders or in association with bladder carcinomas, also related to FGFR3 and PIK3CA mutations. Mosaic mutations of these genes have been occasionally evidenced in syndromic EN. In this report, we describe the cooccurrence of EN, rhabdomyosarcoma, polycystic kidneys and growth retardation in an infant. An oncogenic G12D KRAS mutation was detected in both the epidermal component of the EN and in the rhabodmyosarcoma but neither in the dermal component of the EN lesion nor in unaffected tissues including normal skin or blood. This report identifies for the first time that a KRAS mutation in epiderma causes EN. The observation of the same G12D KRAS mutation in two distinct regions of the body strongly suggests a somatic mosaicism. Finally, this report highlights the potentially underestimated importance of mosaic oncogenes mutations in childhood cancers.

Abstract word count: 182 
Epidermal naevus (EN) is a congenital benign acanthotic skin tumour that presents at birth as a localized epidermal thickening with hyperpigmentation. It usually follows a Blaschko's line and is always diagnosed within the first days to months of life. Histologic examination reveals acanthosis, hyperkeratosis and papillomatosis. $\underline{\text { Somatic mutations of the }}$ FGFR3 gene are the causative lesion of several benign skin tumours first shown in seborrheic keratosis [1] and subsequently in EN [2]. Interestingly, FGFR3 mutations identified in EN are mostly R248C substitutions, which were previously reported as fully oncogenic variants in malignant tumors ([3], review in [4]). Activating PIK3CA mutations are also detected in one third of patients, either alone or in association with FGFR3 alterations [5]. Unlike FGFR3, the main PIK3CA variants observed in EN (E545G) differ from those reported in malignant lesions (E545K, E542K or H1047R) and may present a weaker kinase activity (review in [4]). Finally, no mutation is identified in more than half of the cases, indicating that other causative lesions remain to be identified.

Epidermal nevus can occasionally be associated with complex developmental abnormalities in so called epidermal nevus syndromes [6]. It is also observed in the Proteus syndrome which is related to deleterious PTEN mutations that enhance the PIK3CA activity [7, 8]. Germline mutations in PTPN11, KRAS and BRAF resulting in Noonan (NS) and Cardio-facio-cutaneous (CFC) syndromes also predispose to epidermal hyperplasia and acanthosis $[9,10]$. Similarly, epidermal hyperplasia, acanthosis and epidermal nevi are also reported in Costello syndrome (CS) [11], a congenital developmental disorder due to germline HRAS mutations and characterized by cardiac malformation, short stature, failure to thrive, feeding difficulties, facial dysmorphy, and predisposition to cancers such as early rhabdomyosarcomas, neuroblastomas and transitional bladder carcinomas $[11,12]$. Since CS, CFC and NS are all due to a constitutive activation of the RAS-RAF cascade, this signaling, 
together with FGFR3 and PIK3CA, most likely plays a major role in epithelium growth control.

We now report on an infant showing a large hyperkeratotic lesion present at birth and following a Blaschko's line, fully consistent with the diagnosis of EN (Figure 1A). The child was born with normal weight, stature and head circumference $(2.890 \mathrm{~g}, 49 \mathrm{~cm}$ and $33 \mathrm{~cm}$ respectively) after an unremarkable pregnancy. She had no dysmorphy. The biopsy of the epidermal lesion confirmed the diagnosis by showing hyperkeratosis, acanthosis and papillomatosis (Figure 1B). At 6 months of age, the girl developed an utero-vaginal rhabdomyosarcoma with both embryonal and alveolar phenotypes (Figure 1E). Surprisingly, the abdominal CT-san performed at time of the tumor diagnosis revealed bilateral micropolystic kidneys, a feature that was not found in the mother (no data from the father) (Figure 1D). Moreover, the child presented failure to thrive (short stature -2DS, low weight < -2DS) linked to uncommon feeding difficulties that sustained after the end of chemotherapy. No organic cause of this growth defect was found, in particular the renal function was normal and no sign of intestinal malabsorption was detected. Skeleton X-ray was normal, the echocardiography revealed no malformation. No developmental delay was noticed

The unusual association of several clinical abnormalities prompted us to explore further the genetics of the epidermal nevus and the rhabdomyosarcoma. Neither PIK3CA nor FGFR3 mutations were found in the DNA extracted from the nevus biopsy (Sanger method and SNaPshot methods, respectively). Considering that the tumor was of epithelial origin, we microdissected the hyperproliferative epithelium from the dermal tissue by using laser capture-microdissection (Leica system, Figure 2A and C). In order to search for genetic lesion possibly causative for EN, DNA from the epidermal part was amplified by whole genomic amplification (phi29 DNA polymerase, New England Biolabs); array-CGH was then 
performed, using the oligonucleotide Nimbelgen array-CGH platform. As shown in Figure $1 \mathrm{C}$, no copy number alteration was evidenced.

Despite the presence of an alveolar component, no FKHR translocation was evidenced in the cDNA from the rhabdomyosarcoma. To more thoroughly characterize this atypical mixed tumour, genomic DNA was extracted from the primary biopsy and analyzed by arrayCGH. This analysis showed a gain of whole chromosome 8, an abnormality commonly observed in embryonnal rhabdomyosarcomas, and other less common alterations (Figure 1F).

Given the coexistence of four rare symptoms, i.e. rhabdomyosarcoma, epidermal nevus, micropolystic kidneys, and failure to thrive with feeding difficulties, we postulated that the child may bare a single genetic lesion likely to account for the whole clinical phenotype. Knowing that i) the mutations observed in epidermal nevi lead to a constitutive activation of the RAS-RAF cascade, ii) mutations in the RAS pathway are occasionally detected in rhabdomyosarcomas, and iii) germline mutations in the RAS pathway predispose to both epidermal hyperproliferation and rhabdomyosarcomas, we explored this pathway by gene sequencing. Interestingly enough, whereas no mutation was found in NRAS nor $H R A S$, an oncogenic G12D KRAS mutation was evidenced in both the rhabdomyosarcoma and the epidermal component of the nevus (Figure 2B and 2D). In contrast, no mutation was found in the dermal component of the skin lesion (Figure 2D), nor in a normal skin area; wild-type sequence was also observed in blood lymphocytes, cheeck swab and bone trephine biopsies. No tissue was available to explore the status of KRAS in the polycystic kidney. To sensitize the detection of the G12D mutation, an allele specific PCR was performed according to previously published methods [13]. No variant was detected in the blood, bone biopsy and normal skin. 
This report indicates for the first time that oncogenic KRAS mutation causes EN. Mutations in the RAS pathways are found in approximately $15 \%$ of RMS $[14,15,16]$; hence, the KRAS mutation in the malignant tumour could be more expected. However, most of the mutations involve the NRAS codon 61 and no mutation in KRAS has been observed in the two most recent series of 31 and 30 primary rhabdomyosarcomas $[14,15]$. Only one mutation in $K R A S$ codon 12 has been reported in the whole literature [16]. Although $K R A S$ mutation has not been involved in polycystic kidney in human, it is noteworthy that, in the mouse, selective hyperexpression of Ras in developing renal tubules results in the development of multiple renal cysts [17]. This experimental observation provides strong support in favor of a causative role of the KRAS mutation in our patient's renal malformation.

Although we can not formally rule out that the two identical KRAS alterations occur

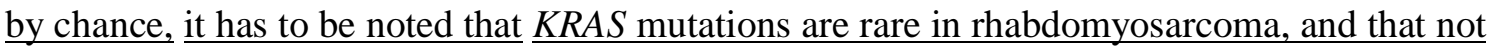
a single G12D KRAS mutation in rhabdomyosarcoma is reported in the COSMIC cancer mutation database. Moreover, the whole clinical phenotype is consistent with a broad hyperactivation of the Ras pathway. Altogether, these data rather suggest a mosaicism than an isolated and independent occurrence of the two mutations. This mutation is present in both mesodermally and ectodermally derived tissues, suggesting that it may have occur relatively early during development. Consistently, previous reports support the hypothesis of oncogenic mosaic mutation being responsible for EN. First, FGFR3 and PIK3CA mutations observed in the abnormal Blaschko's line are systematically absent from the normal skin counterpart; this has for long suggested that EN results from somatic mutations occurring in a metameric pattern during development and leading to a mosaicism [2, 5]. Hernandez et al reported several patients baring multiple EN with the same FGFR3 R248C mutation, but without the severe achondroplastic phenotype that is usually associated with R248C germline mutations [18]. Of even greater interest, an undoubtful mosaic FGFR3 mutation has been reported in a 
patient showing multiple EN, seizures and mental retardation [6]. The higher risk of cancer conferred by mosaic mutations in FGFR3 or PIK3CA is all the more plausible that several cases of young patients harboring both EN and low-grade bladder cancers have been reported (case report and review in [19]). To our knowledge, rhabdomyosarcoma has been associated with EN in only two patients [7, 20]. However, as mentioned above, the constitutive activation of RAS pathway, characteristic of CS, predisposes to both rhabdomyosarcomas and hyperproliferative skin disorders $[12,21]$. Noticeably, the patient of our present report shows a clinical phenotype that can be partially related to $\mathrm{CS}$, since growth defect, feeding difficulties and rhabdomyosarcoma are frequent features of CS. On a molecular point of view, the present case could also be related to a mosaic form of NS or CFC, a proportion of which are characterized by germline $K R A S$ mutations and occasionally associated with epidermal naevus. However, the germline mutations reported in CFC and NS almost never involve codons 12,13 or 61 (review in $[22,23]$ ) since only one CFC patient with a KRAS G12S mutation has been reported [24]. Analyses of the functional consequences of $K R A S$ variants responsible for NS and CFC demonstrate their obvious property to hyper-activate the RAS signaling, that is nevertheless less pronounced than the one mediated by the G12D variant [9, 10]. Of note, ubiquitous expression of G12D K-ras mutant in mice leads to early embryonic lethality [25]. Altogether, KRAS activating germline mutations responsible for NS and CFC might remain viable because KRAS activity is lower than that of G12D variant. The present report suggests that widespread KRAS G12D mutation remains compatible with life provided nevertheless that it is restricted to a specific mosaic pattern.

Segmental Neurofibromatosis type 1 is a well-known cancer-prone developmental disorder due to a mosaic NF1 mutation that leads to a RAS hyperactivation in a specific pattern of tissues. Likewise, mosaicism in HRAS has been described in Costello patients [26]. Our present report describes a comparable genetic abnormality in the RAS pathway that 
results in a new developmental disorder with cancer predisposition. This observation of segmental G12D KRAS mutation highlights the potentially underestimated importance of oncogenes mosaic mutations in childhood cancers. 
Acknowledgement: we thank Dr Daniel Orbach, Dr Françoise Boman, Pr JeanFrançois Mercier, Pr Jean-Pierre Hugo for their kind help in the care of the child and for providing the biological samples. We thank Dr Helene Cave for her kind advice in reviewing the manuscript. This work was supported by grants from the INCa (plateforme de génétique moléculaire des cancers) and the Ligue Nationale Contre le Cancer (Equipes labellisées: FB, OD and AH, FR). 
Legend to figures

Figure 1: Phenotype and copy number analysis of the two tumours:

1A: Picture showing the epidermal nevus following the Blaschko line.

1B: Hematoxylin Eosin Safran (HES) staining of the biopsy of the nevus: hyperkeratosis (h), acanthosis (a) and papillomatosis (p).

1C: array-CGH performed on Nimbelgen oligonucleotide array, using 50ng DNA extracted from micro-dissected samples of the epidermal component of the biopsy after whole genome amplification with phi29 DNA polymerase. Reference DNA was amplified according to the same procedures. No alteration is evidenced.

1D: Abdominal CT-scan showing the huge utero-vaginal rhabdomyoasarcoma $(\rightarrow)$ and the micropolycystic kidney $(\longrightarrow)$.

1E: HES of the tumour biopsy in its alveolar sub-component.

1F: array-CGH analysis of DNA from the tumor biopsy: gain of whole chromosomes $2,4,8$, 12,13 ; amplifications $(\rightarrow)$ at 19p13.2, 19q13.2 and 22q11.21.

Figure 2: G12D KRAS mutation in the epidermal component of the lesion.

1A: HES on the whole epidermal nevus biopsy; e: epidermal part of the lesion; d: dermal component.

1B: exon1 KRAS sequencing shows an unbalanced proportion of the normal allele and the G12D variant in DNA extracted from the whole section of the biopsy 
1C: laser capture micro-dissection using Leica microcapture system. The epidermal cells are isolated from the dermal tissue. After micro-dissection, the samples were pellet in Qiagen buffer; DNA was extracted with QiaAmp DNA micro-kit (Qiagen). 20 ng were used for direct PCR amplification and sequencing; 10ng were amplified with phi29 DNA polymerase for subsequent array-CGH.

1D: KRAS sequencing in the micro-dissected epidermal (e) and dermal (d) tissues. In the purely epidermal component, sequencing shows the heterozygous G12D mutation with a balanced peak for both normal and variant alleles. In contrast, no mutation is evidenced in the pure dermal component. 
References

1 Logie A, Dunois-Larde C, Rosty C, et al. Activating mutations of the tyrosine kinase receptor FGFR3 are associated with benign skin tumors in mice and humans. Hum Mol Genet 2005;14(9):1153-60.

2 Hafner C, van Oers JM, Vogt T, et al. Mosaicism of activating FGFR3 mutations in human skin causes epidermal nevi. J Clin Invest 2006;116(8):2201-7.

3 Cappellen D, De Oliveira C, Ricol D, et al. Frequent activating mutations of FGFR3 in human bladder and cervix carcinomas. Nat Genet 1999;23(1):18-20.

4 Toll A, Real FX. Somatic oncogenic mutations, benign skin lesions and cancer progression: where to look next? Cell Cycle 2008;7(17):2674-81.

$5 \quad$ Hafner C, Lopez-Knowles E, Luis NM, et al. Oncogenic PIK3CA mutations occur in epidermal nevi and seborrheic keratoses with a characteristic mutation pattern. Proc Natl Acad Sci U S A 2007;104(33):13450-4.

6 Garcia-Vargas A, Hafner C, Perez-Rodriguez AG, et al. An epidermal nevus syndrome with cerebral involvement caused by a mosaic FGFR3 mutation. Am J Med Genet $A$ 2008;146A(17):2275-9.

7 Vidaurri-de la Cruz H, Tamayo-Sanchez L, Duran-McKinster C, et al. Epidermal nevus syndromes: clinical findings in 35 patients. Pediatr Dermatol 2004;21(4):432-9.

8 Loffeld A, McLellan NJ, Cole T, et al. Epidermal naevus in Proteus syndrome showing loss of heterozygosity for an inherited PTEN mutation. Br J Dermatol 2006;154(6):1194-8.

9 Niihori T, Aoki $Y$, Narumi $Y$, et al. Germline KRAS and BRAF mutations in cardio-faciocutaneous syndrome. Nat Genet 2006;38(3):294-6.

10 Schubbert S, Zenker M, Rowe SL, et al. Germline KRAS mutations cause Noonan syndrome. Nat Genet 2006;38(3):331-6.

11 Nguyen V, Buka RL, Roberts BJ, et al. Cutaneous manifestations of Costello syndrome. Int J Dermatol 2007;46(1):72-6.

12 Aoki $\mathrm{Y}$, Niihori $\mathrm{T}$, Kawame $\mathrm{H}$, et al. Germline mutations in HRAS proto-oncogene cause Costello syndrome. Nat Genet 2005;37(10):1038-40.

13 Case $M$, Matheson $E$, Minto $L$, et al. Mutation of genes affecting the RAS pathway is common in childhood acute lymphoblastic leukemia. Cancer Res 2008;68(16):6803-9.

14 Chen Y, Takita J, Hiwatari M, et al. Mutations of the PTPN11 and RAS genes in rhabdomyosarcoma and pediatric hematological malignancies. Genes Chromosomes Cancer 2006;45(6):583-91.

15 Martinelli S, McDowell HP, Vigne SD, et al. RAS signaling dysregulation in human embryonal Rhabdomyosarcoma. Genes Chromosomes Cancer 2009;48(11):975-82.

16 Stratton MR, Fisher C, Gusterson BA, et al. Detection of point mutations in N-ras and K-ras genes of human embryonal rhabdomyosarcomas using oligonucleotide probes and the polymerase chain reaction. Cancer Res 1989;49(22):6324-7.

17 Schaffner DL, Barrios R, Massey C, et al. Targeting of the rasT24 oncogene to the proximal convoluted tubules in transgenic mice results in hyperplasia and polycystic kidneys. Am J Pathol 1993;142(4):1051-60.

18 Hernandez S, Toll A, Baselga E, et al. Fibroblast growth factor receptor 3 mutations in epidermal nevi and associated low grade bladder tumors. I Invest Dermatol 2007;127(7):1664-6.

19 Garcia de Jalon A, Azua-Romeo J, Trivez MA, et al. Epidermal naevus syndrome (Solomon's syndrome) associated with bladder cancer in a 20-year-old female. Scand J Urol Nephrol 2004;38(1):85-7.

20 Rongioletti $F$, Rebora A. Epidermal nevus with transitional cell carcinomas of the urinary tract. J Am Acad Dermatol 1991;25(5 Pt 1):856-8. 
21 Gripp KW. Tumor predisposition in Costello syndrome. Am J Med Genet C Semin Med Genet 2005;137C(1):72-7.

22 Aoki $Y$, Niihori T, Narumi $Y$, et al. The RAS/MAPK syndromes: novel roles of the RAS pathway in human genetic disorders. Hum Mutat 2008;29(8):992-1006.

23 Gelb BD, Tartaglia M. Noonan syndrome and related disorders: dysregulated RAS-mitogen activated protein kinase signal transduction. Hum Mol Genet 2006;15 Spec No 2:R220-6.

24 Nava C, Hanna N, Michot C, et al. Cardio-facio-cutaneous and Noonan syndromes due to mutations in the RAS/MAPK signalling pathway: genotype-phenotype relationships and overlap with Costello syndrome. J Med Genet 2007;44(12):763-71.

25 Tuveson DA, Shaw AT, Willis NA, et al. Endogenous oncogenic K-ras(G12D) stimulates proliferation and widespread neoplastic and developmental defects. Cancer Cell 2004;5(4):375-87.

26 Gripp KW, Stabley DL, Nicholson L, et al. Somatic mosaicism for an HRAS mutation causes Costello syndrome. Am J Med Genet A 2006;140(20):2163-9. 
The Corresponding Author has the right to grant on behalf of all authors and does grant on behalf of all authors, an exclusive licence (or non exclusive for government employees) on a worldwide basis to the BMJ Publishing Group Ltd to permit this article (if accepted) to be published in JMG and any other BMJPGL products and sublicences such use and exploit all subsidiary rights, as set out in our licence.

Competing Interest: None declared 

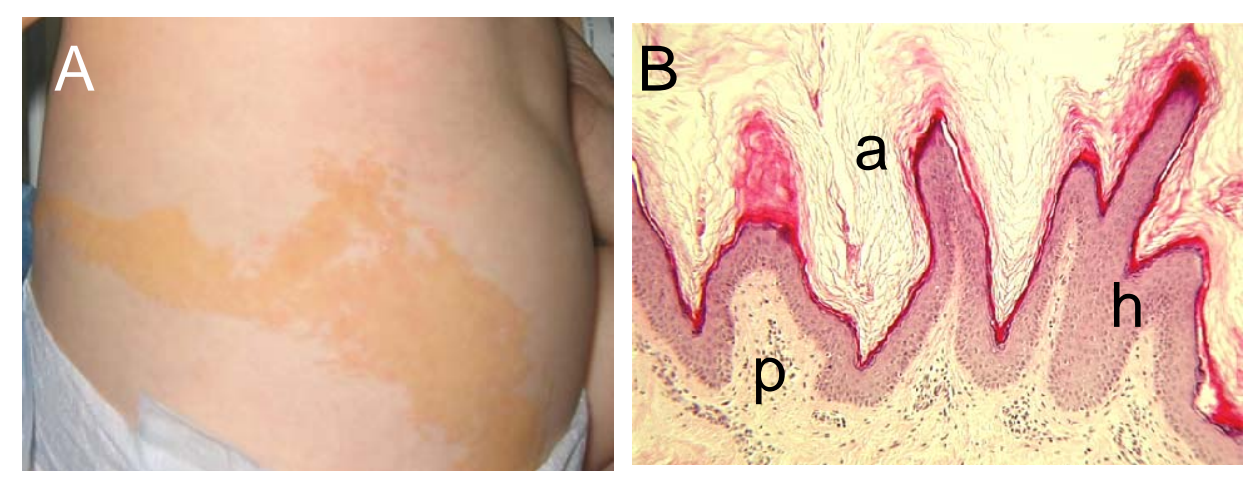

C
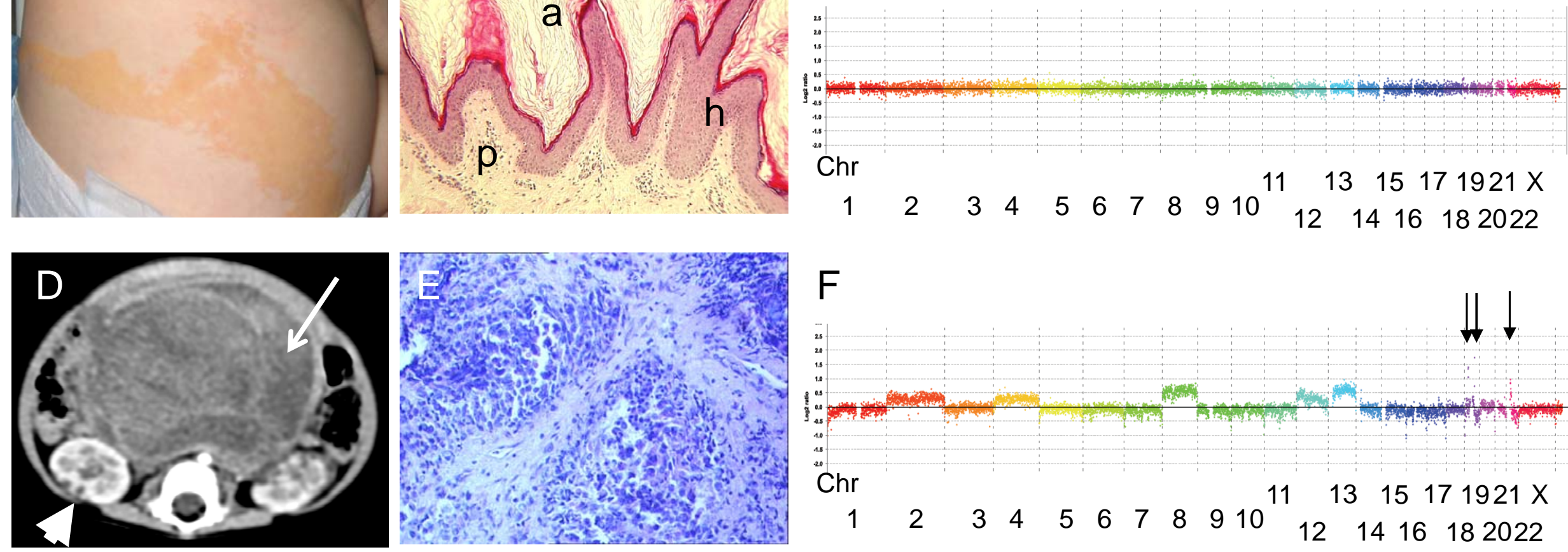


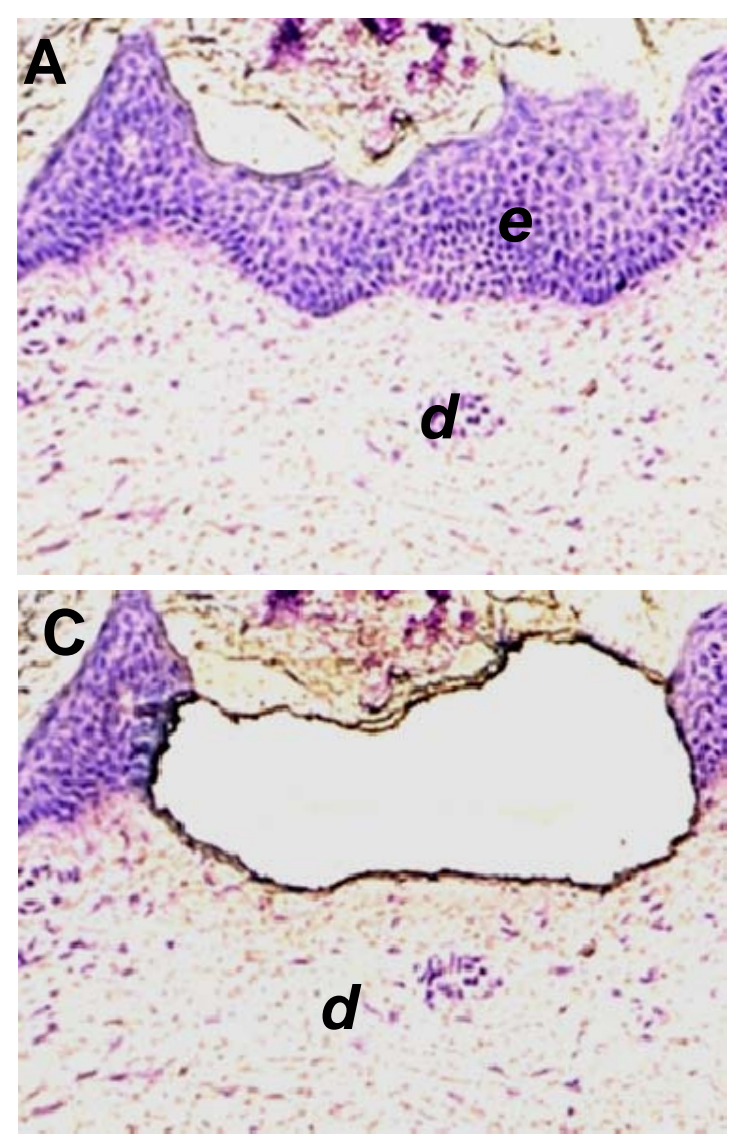

B

TG G A C T G F T G G G TA C

$T G G A G C T G F T G G C G T A C$

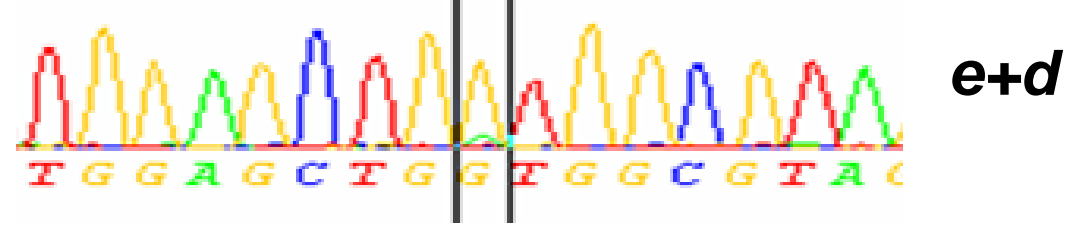

D IGGAGCTGRIGGCGTA

$\Gamma G G A G C T G R T G G C G T A \mid e$

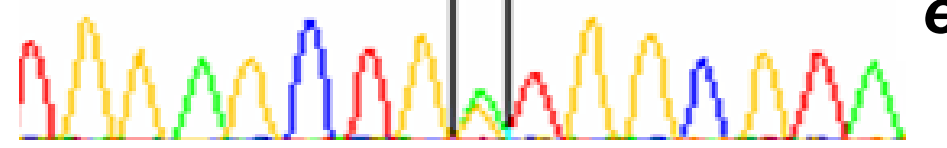

$T G G A G C T G|F| T G C G T A$

$T G G A G C T G F T G G C G T A$

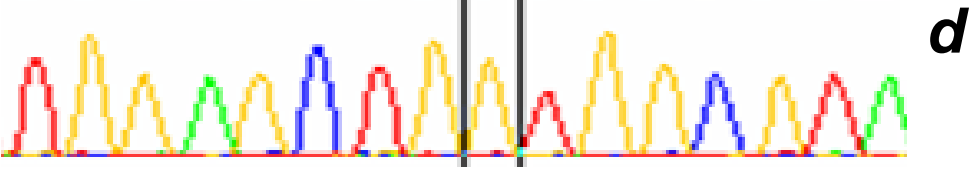

